\title{
Outreach Librarian to Black Students: A De Facto Role
}

\author{
Katrina Spencer \\ Middlebury College
}

Abstract: While some predominantly white institutions can offer unique and laudable educational experiences to Black college students, they can also lack resources that provide these same students with a culturally affirming milieu. Black faculty and staff can organize their time, energy and resources to offer culturally enriching experiences for Black students. However, this additional labor is not systematically seen, cataloged or recognized as a core or essential duty. The author describes some effective forms of outreach from her personal experience and simultaneously asks whose job it is to meet the cultural needs of this population.

Keywords: outreach, innovation, Black library users, higher education

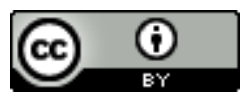

This is an Open Access article distributed under the terms of the Creative Commons Attribution 4.0 International License (http://creativecommons.org/licenses/by/4.0), which permits unrestricted use, distribution, and reproduction in any medium, provided the original work is properly cited. 
Studying in rural New England offers fewer opportunities for cultural affirmation, engagement and celebration for Black college students than places like Atlanta, Chicago, Philadelphia and Washington, D.C. Much of this is a numbers game: less than $1.5 \%$ of the population in Middlebury, Vermont is Black. So access to certain amenities like getting one's hair regularly braided or faded is a quest unto itself, not to mention getting access to a slew of other cultural practices and traditions. As one of the few Black staff people at Middlebury College, a small, private, liberal arts institution, I try to create opportunities to celebrate Blackness where and when I can. However, one of my ongoing, professional challenges is remembering that my primary role on this campus is not in academic affairs, residential life or counseling; rather, it's in the library and in the promotion of information literacy. So when I begin to craft these events and experiences, I must ask myself several questions: "As a result of this effort, are students learning more about library use and how to seek and use information? Is this an appropriate effort for a librarian to lead? Is this an appropriate use of my work time?" While a number of my edifying ideas do notpass this test, let me tell you about three efforts that responded to these questions with a clear and resounding, "Yes!" 

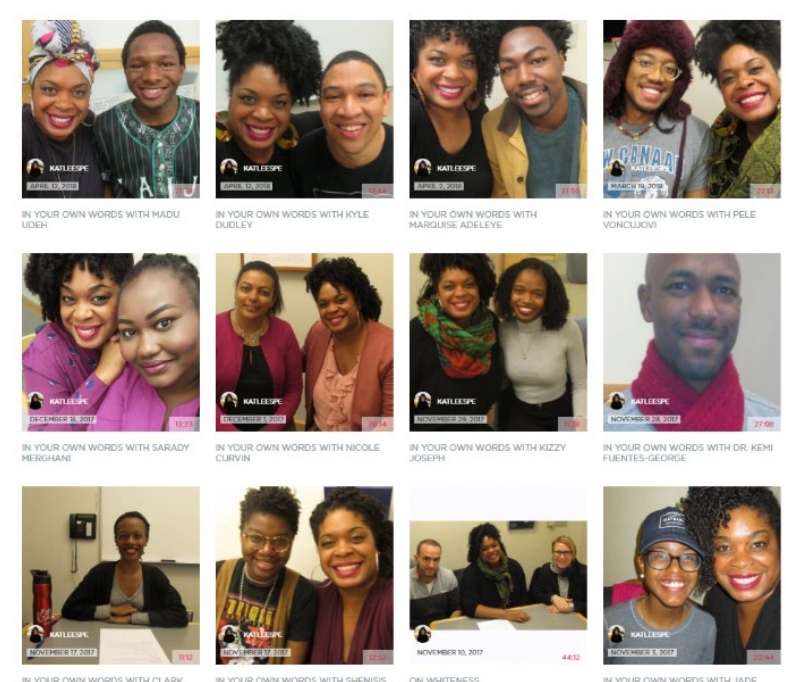

Image 1: $\underline{\text { In Your Own Words }}$

Inspired by University of South Carolina professor Dr. Nicole Cooke's pedagogies, I encouraged Black students, staff and faculty to share their experiences and perceptions of race in voluntary, recorded interviews that would be archived in Special Collections, with StoryCorps and through the Library of Congress. They were also aired on our campus' radio station. While I lovingly hold onto the term "Black" as an identifier, I realize how it can be limiting as a blanket term when referring to both the African Americans from the Southside of Chicago-- children of the Mass Migration-- and one of my library users who is the first generation daughter of Sudanese immigrants who practice Islam. This is exactly what I wanted to explore with these recordings: the inherent diversity sometimes cloaked by the term "Black." In order to highlight the nuances, community members shared their impressions, stories and understandings in their own words. Some of the questions posed to them were "How has your understanding of racial and ethnic identity evolved over time?," "How do notions of race and ethnicity change based on where you are and who you're with?" and "What do you wish others knew about race and ethnicity?" Our collections now have an archive of these interviews that serve as a small 
snapshot of racial discourse on the Middlebury campus at a particular time. Moreover, participants learned that they are not only consumers of knowledge, they are creators, too.

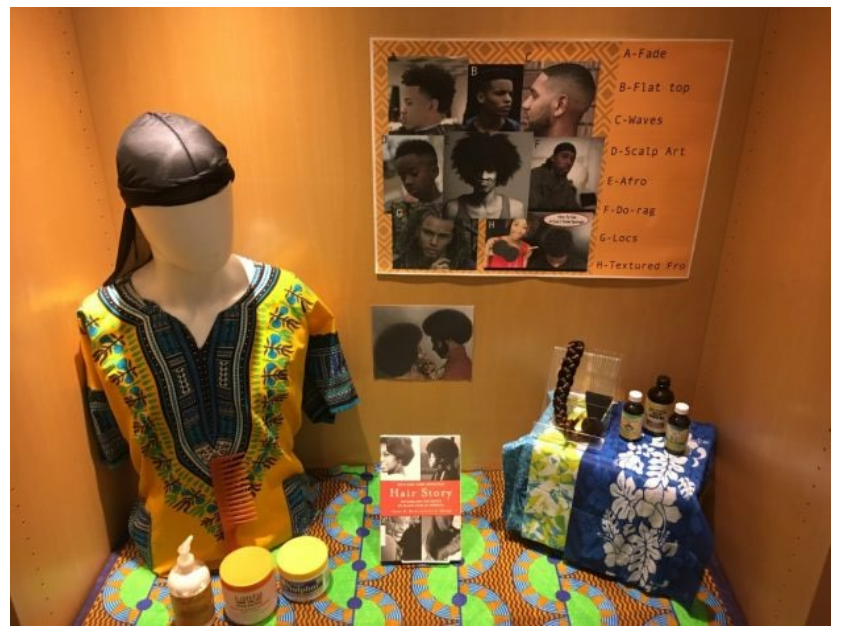

Image 2:

In small, predominantly white towns such as this, many Black people lament the inability to seek appropriately skilled haircare from professionals with experience in caring for our textures of hair. Students rely on each other for a variety of styles or take a bus to Burlington, about an hour away. In these communities, only some residents know of the extra efforts Black people make to seek out haircare. To highlight this narrative and the history surrounding haircare traditions of the Black diaspora, a group of students, staff, faculty and community members put together an exhibit that showcased the evolution of Black hair care traditions from around the world. With creams, butters and oils, combs, razors and flat irons, extensions, wigs and doo rags, we used library spaces to exhibit the great diversity of expression represented in and by our hair. Students learned to build our collections, to reserve library spaces for creative use and to discover resources by using controlled vocabularies. A rumor existed that students of color used library spaces less often than their white peers. What I have learned is that if Black students are given a reason to engage with these spaces, they will. It helps, no 
doubt, if they have a relationship of trust with and guidance from a staff or faculty member who looks like them and shares some of their cultural perspectives, experiences and background.

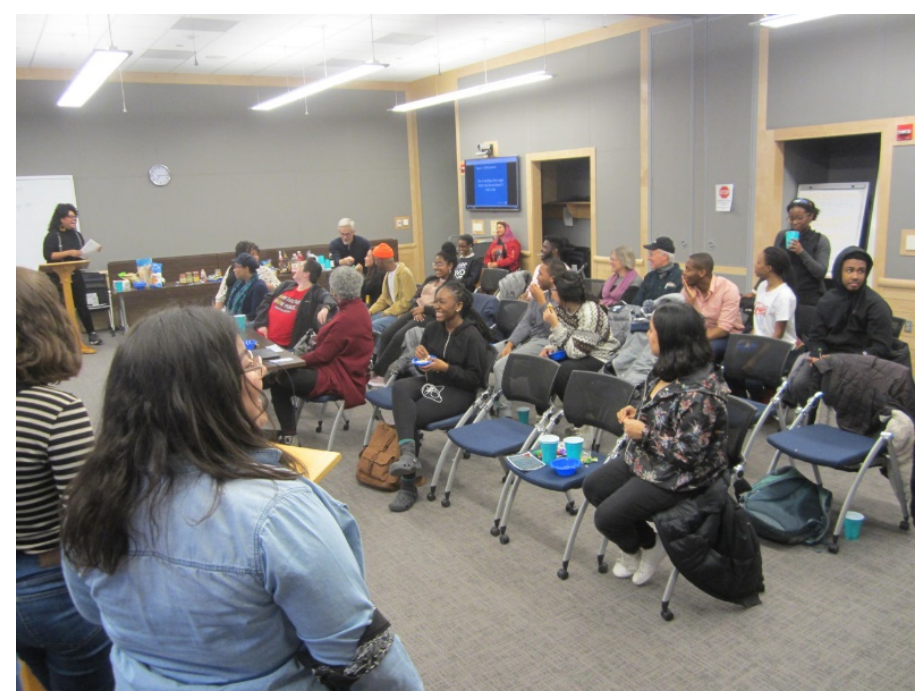

Image 3: Black History Month Jeopardy

The year 2020 will be the third that I work with a broad array of community members to create the Black History Month Jeopardy event. Following Alex Trebek's television show format quite closely, students, staff, faculty and community members gather for one evening in February to compete in multiple rounds of trivia that are thematically centered on Black people(s). Categories include public figures in politics, journalism, literature, science, television/cinema/Hollywood and sports; famous quotations and song lyrics; geography; the library's collections and more. Many of us schooled at predominantly white institutions (PWIs) realize that the curricula we were exposed to did not prioritize Black people's contributions to history, art, literature, science or a variety of other disciplines. Moreover, in order to learn about the deprioritized contributions, Black people(s) have been obligated to create and pursue a separate curriculum in addition to the formal one prepared for us in class. This event allows the library to showcase its wares, to publicly value a variety of epistemologies and to 
provide space to community members as we celebrate information that has long been suppressed.

With this event, participants are also afforded an opportunity to interrogate what knowledge is centered and prized on a college campus and to ask why. But, perhaps most importantly, they can ask, "If my story is not being told here, what can I do about it?"

These three outreach efforts have allowed me as library staff to proactively seek out Black peoples' expertise, narratives and engagement within library spaces, with collections and with several information literacy tasks. Is the work "easy"? Not necessarily. It takes a good deal of scheduling, coordination and planning. Is it worthwhile? Absolutely. One of the signs that you're doing something right as a librarian is that you have repeat "customers" and I find that once a connection is made, I see the same people seeking more from me. They have developed a culture of expectation and query me about what's next on the calendar. Is this my job? That part is harder to answer. It is certainly my job to reach library users and help them to understand that the library can be adapted to their needs. However, there is always a competition for my attention and that has been one of the hardest balancing acts I've ever performed. While my job description says I am on the Research and Instruction team, with core responsibilities in teaching, consultations, maintenance of research guides and some collection development, I suspect that my most impactful work is done in outreach and engagement. Moreover, when it comes to outreach to racially diverse groups, as the only Black employee in the library system, the work proves challenging in its equal distribution. Perhaps the answer to the question is that this is everyone's job-- but who is actually available and able to do it effectively? And how is it monitored and sustained as everyone's responsibility? I'll certainly continue to weigh this topic as I progress on my career trajectory. If you, too, are straddling this line as a de facto outreach librarian to underrepresented library users, comment below and let us know how you manage. 